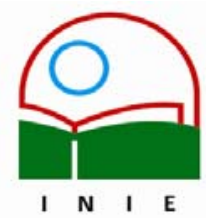

Actualidades Investigativas en Educación

Revista Electrónica publicada por el

Instituto de Investigación en Educación

Universidad de Costa Rica

ISSN 1409-4703

http://revista.inie.ucr.ac.cr

COSTA RICA

\title{
FUNDAMENTOS TEÓRICOS QUE SUSTENTAN LA METODOLOGÍA PARA EL DESARROLLO DE LAS HABILIDADES DE EXPRESIÓN GRÁFICA EN LOS EGRESADOS EN INGENIERÍA AGRONÓMICA
}

THEORETICAL FUNDAMENTALS THAT SUSTAIN METHODOLOGY FOR DEVELOPMENT OF THE SKILLS OF EXPRESSION IN FIGURE GRADUATES IN AGRICULTURAL ENGINEERING

Volumen 8 , Número 3

pp. 1-18

Este número se publicó el 15 de diciembre 2008

Oscar Paz Gómez

La revista está indexada en los directorios:

LATINDEX, REDALYC, IRESIE, CLASE, DIALNET, DOAJ, E-REVIST@S,

La revista está incluida en los sitios:

REDIE, RINACE, OEI, MAESTROTECA, HUASCARAN

Los contenidos de este artículo están bajo una licencia Creative Commons 


\title{
FUNDAMENTOS TEÓRICOS QUE SUSTENTAN LA METODOLOGÍA PARA EL DESARROLLO DE LAS HABILIDADES DE EXPRESIÓN GRÁFICA EN LOS EGRESADOS EN INGENIERÍA AGRONÓMICA
}
THEORETICAL FUNDAMENTALS THAT SUSTAIN METHODOLOGY FOR DEVELOPMENT OF THE SKILLS OF EXPRESSION IN FIGURE GRADUATES IN AGRICULTURAL ENGINEERING

\begin{abstract}
Oscar Paz Gómez ${ }^{1}$
Resumen: Una de las problemáticas que más está afectando a los graduados de la Ingeniería Agronómica en Cuba, en la actualidad, es el insuficiente desarrollo de las habilidades de representación, lectura e interpretación de documentación técnica gráfica por el ingeniero agrónomo actual, cuyas causas aparentes están dadas por el gran volumen de contenido a impartir en las asignaturas de Expresión Gráfica y el poco tiempo de actividad docente presencial con los alumnos. Otro aspecto a considerar es que los métodos y medios de enseñanza aprendizaje utilizados no están respondiendo a la dinámica actual de este proceso de enseñanza - aprendizaje.
\end{abstract}

Palabras clave: METODOLOGÍA Y FUNDAMENTOS TEÓRICOS

Abstract: One of these problems that is most affecting to the graduates of the Agronomy Engineering in Cuba, at the present time, is the insufficient development of the representation abilities, reading and interpretation of documentation technical graph for the current agricultural engineer whose apparent causes are given by the great content volume to impart in the subjects of Graphic Expression and the little time of present educational activity with the students. Another aspect to consider is that the methods and teaching means - used learning is not responding to the current dynamics of this teaching process - learning.

Key Word: METHODOLOGY AND THEORETICAL FOUNDATIONS

\section{Introducción}

El análisis dialéctico - materialista de todo objeto o fenómeno plantea la necesidad de estudio en el proceso de su desarrollo. Por ello, para conocer en toda su magnitud las exigencias que en la actualidad tiene la formación de profesionales en Ingeniería Agronómica en Cuba es importante precisar los problemas generales básicos de la producción y los servicios correspondientes a esta profesión y a cuya solución debe estar dirigida la formación del graduado.

\footnotetext{
${ }^{1}$ Máster en Educación Superior de la Universidad de Ciego de Avila, Cuba. Profesor del Centro de Estudios Hidrotécnicos, Facultad de Ingeniería de la Universidad de Ciego de Avila, Cuba. Ha ofrecido el curso de Metodología de la Investigación Educativa en un Diplomado sobre Educación ofertado por ésta universidad a la Universidad de Córdoba, Colombia. Ha participado en 17 eventos de Ingeniería Gráfica y Pedagogía y ha escrito 15 artículos científicos sobre estas temáticas.
}

Dirección electrónica: oscarp@ingenieria.unica.cu

Artículo recibido: 14 de julio, 2008

Aprobado: 4 de diciembre, 2008

Volumen 8, Número 3, Año 2008, ISSN 1409-4703 
La formación de profesionales en Ingeniería Agronómica, que garanticen un desarrollo sostenible en nuestro país, ha sido una preocupación constante del sistema de Educación Superior en Cuba. El perfeccionamiento del proceso de enseñanza - aprendizaje para la formación cada vez más competente de este tipo de profesional ha sido una realidad, pero aún, persisten insuficiencias todavía en dicha formación, siendo considerablemente significativas las relacionadas al insuficiente desarrollo de las habilidades de representación (dibujo), lectura e interpretación de planos topográficos por el Ingeniero Agrónomo actual.

\section{Fundamentación del problema}

Tomando como premisa las dificultades de los alumnos de la carrera de Ingeniería Agronómica de la Universidad de Ciego de Ávila, Cuba, en la asignatura Dibujo - Topografía, en cuanto a la representación, lectura e interpretación de planos topográficos, cuestión que se manifiesta en la discusión del trabajo final integrador de la asignatura, así como posteriormente en la solución de tareas técnicas de las asignaturas Riego y Drenaje y de Ciencias de Suelos (asignaturas a las cuales le aporta el contenido de la asignatura Dibujo Topografía),. Además, profesionales de la producción agropecuaria del país han manifestado que existen dificultades con los graduados de la carrera de Agronomía en cuanto a la lectura e interpretación de planos de suelos y de usos generales, lo mismo ocurre con los cartogramas agroquímicos. Esto pone de manifiesto que el alumno antes de graduarse como profesional en Ingeniería Agronómica, no ha desarrollado eficientemente las habilidades de representación, lectura e interpretación de los planos topográficos.

En encuestas realizada a docentes de otras Universidades del país como lo son: la Universidad de Matanzas, la Universidad de las Tunas, la Universidad Agraria de la Habana, etc., existen también estas dificultades con los graduados en Ingeniería Agronómica de estas provincias.

Se conoce además a través de los diferentes Congresos de Ingeniería Gráfica efectuados en distintos países tales como: España, Brasil, Argentina, México, Cuba, etc., que esta situación se manifiesta en la actualidad en otras ingenierías, incluyendo la Arquitectura.

A partir de lo anteriormente señalado, se procedió a realizar un diagnóstico de las asignaturas Dibujo y Topografía a través de los diferentes programas de estudios por los que ha transitado la Educación Superior en Cuba, lográndose una caracterización histórica del 
sistema de habilidades a formar en los alumnos de Ingeniería Agronómica y especialmente de las habilidades de expresión gráfica. Además, en dicho diagnóstico salieron a la luz un grupo de dificultades en el proceso de enseñanza - aprendizaje de la asignatura Dibujo Topografía que tiene incidencia en el desarrollo más eficiente de las referidas habilidades de expresión gráfica en dichos alumnos en la actualidad. Considera el autor de este trabajo genera las dificultades que se describen a continuación, la primera es la que más incidencia tiene en la mencionada problemática, por lo que es a la que se le presta atención en esta investigación, sin dejar de tener en cuenta las demás dificultades.

\section{Dificultades:}

- Excesivo contenido de la asignatura planificado para la actividad docente presencial, lo que hace que los métodos y medios de enseñanza "tradicionales", utilizados en la mayoría de los CES de enseñaza agropecuaria del país donde se imparte la asignatura Dibujo - Topografía, no responden a la dinámica del proceso de enseñanza aprendizaje de la asignatura

- No todos los centros del país donde se imparte la asignatura Dibujo - Topografía en la carrera de Ingeniería Agronómica lo hacen de forma integrada, pues primero imparten el contenido del Dibujo, y lo hace un docente de Dibujo, y posteriormente imparten el contenido de Topografía y lo hace un docente de Topografía.

- No se realiza un trabajo final integrador de la asignatura en la mayoría de los centros del país con carreras de perfil agropecuario, con excepción de algunos, que lo realizan.

- No se contempla en la asignatura (en la mayoría de los CES de enseñanza agropecuaria del país), la habilidad "lectura", la cual debe preceder a la habilidad interpretación.

- El insuficiente nivel académico de los alumnos que están ingresando a las carreras ingenieriles en los últimos años. En el caso de la carrera de Agronomía se están afectando los niveles de asimilación del contenido de la asignatura Dibujo - Topografía, en dichos estudiantes al recibirla, por esta causa.

Lo hasta aquí descrito pone de manifiesto la existencia de una problemática fáctica que consiste en el insuficiente dominio de las habilidades de representación, lectura e 
interpretación de documentación técnica gráfica por parte de los alumnos de la carrera de Ingeniería de la Universidad de Ciego de Ávila, Cuba.

No existen antecedentes en la enseñanza superior de Cuba de un estudio profundo sobre el insuficiente desarrollo de las habilidades de representación, lectura e interpretación de documentación técnica gráfica en los graduados en Ingeniería Agronómica.

El estudio realizado precisa la búsqueda de una metodología basada en nuevos métodos y medios de enseñanza - aprendizaje capaces de que en menos tiempo de actividad docente presencial se logre una docencia en la asignatura Dibujo - Topografía de alta calidad, que contribuya a satisfacer las necesidades actuales de los graduados en Ingeniería Agronómica en cuanto al desarrollo más eficiente de las habilidades de representación, lectura e interpretación de documentación técnica gráfica. Del mismo modo, constituye una necesidad la sistematización de dichas habilidades, y la definición de las operaciones por realizar en cada una de ellas.

En relación a lo antes expuesto, el objetivo del presente trabajo es argumentar los fundamentos teóricos que sustentan la propuesta metodológica, con vistas a contribuir a satisfacer las necesidades actuales del graduado en Ingeniería Agronómica, en cuanto al desarrollo más eficiente de las habilidades de expresión gráfica.

\section{DESARROLLO}

\section{Fundamentos teóricos que sustentan la metodología que se propone}

La propuesta tiene como base metodológica la concepción materialista y dialéctica del mundo desde la cual se asumen posiciones respecto de los referentes que le sirven de soporte en el plano epistemológico, sociológico, psicológico y pedagógico (Díaz, 2005, pp. 25-26).

La posición epistemológica defiende la asimilación de la cultura por las personas como un proceso de aprendizaje y construcción del conocimiento a partir de la dialéctica entre la teoría y la práctica, en un recorrido de aproximación sucesiva al objeto de estudio hasta su dominio consciente, lo cual constituye un sustento fundamental de la metodología, a partir de la manera que se presenta la ejercitación sistemática de las operaciones de las habilidades y su vínculo con la actividad práctica de su profesión. 
Otra posición de partida es la concepción de la educación como un fenómeno social que no se puede aislar de los contextos en que se desarrolla, desde su necesidad como exigencia de la sociedad, hasta su desarrollo en un marco de relaciones sociales que la caracterizan. El educando en formación interactúa con sus semejantes, con sus docentes, con la familia, con la escuela y con la comunidad, contextos en los que socializa toda su formación, incluyendo sus habilidades profesionales, que a la vez se proyectan como necesidad social, desde la acción social y para su contribución a la sociedad.

En el plano psicológico la metodología se sustenta en el enfoque Histórico Cultural desarrollado por Vigotsky y sus seguidores, específicamente los principios que precisan el papel de la educación como anticipación y estímulo del desarrollo de la personalidad.

Las habilidades para ejecutar el proceso de enseñanza - aprendizaje de la asignatura se asumen como un proceso esencialmente social en el que se resalta la actividad cognoscitiva, en el propio desarrollo de la actividad; esto resulta fundamental para interiorizar la comprensión y se convierte en un proceso intermedio entre la realidad externa y el proceso interno del pensamiento.

Desde el punto de vista pedagógico la metodología se sustenta en la tendencia integradora y contextualizada de los componentes personales y personalizados del proceso docente - educativo en la que precisan todas las relaciones entre los objetivos - contenidos - métodos - medios y evaluación, a partir de una posición del docente como sujeto del proceso de enseñanza - aprendizaje. El docente debe ser creador en el desarrollo curricular y orientador, diseña dicho proceso a partir del diagnóstico, orienta y evalúa en un proceso de comunicación educativa y en el que el alumno también es sujeto del proceso docente educativo en cuanto logra protagonismo en su propio desarrollo y lo hace con conciencia e interviniendo de forma activa a partir de sus intereses, motivaciones y necesidades.

La concepción de objetivo que se asume, es integradora, como el componente rector del proceso de enseñanza - aprendizaje, que precisa la máxima aspiración formativa y en el que se revelan los elementos instructivos, educativos y desarrolladores de la personalidad de los educandos en formación para el desarrollo de las habilidades de expresión gráfica en el proceso enseñanza - aprendizaje de la asignatura.

El contenido de la enseñanza está determinado en la metodología por las invariantes funcionales que deben asimilar los educandos en formación para apropiarse de las habilidades de expresión gráfica en el proceso enseñanza - aprendizaje, en relación con 
todo el contenido conceptual, procedimental y actitudinal a desarrollar con los alumnos del segundo año de Ingeniería Agronómica.

Se precisan los métodos y procedimientos que debe ejecutar el docente que dirige el proceso de enseñanza - aprendizaje y los que debe ejecutar el alumno en formación, para contribuir desde el sistema de tareas docentes de la asignatura en integración entre los temas que la conforman, a un desarrollo más eficiente de las habilidades de expresión gráfica.

La propuesta utiliza los principios del "Enfoque Histórico Cultural" y de la actividad al aplicar el sistema de principios didácticos sobre los que se ha de llevar a cabo la enseñanza basada en sus concepciones teóricas. Se propone además como alternativa didáctica factible, una estructura del proceso de enseñanza - aprendizaje de la asignatura a partir de considerar los modelos específicos planteados por Nina F. Talízina (Pérez, 1998: 42) que garantiza la cientificidad del proceso de enseñanza - aprendizaje, profundizando específicamente en el modelo del proceso. El mismo consta:

\section{a) Modelo de los objetivos}

De acuerdo con este modelo, los objetivos se presentan en forma de tareas típicas, gracias a los cuales se organiza el aprendizaje, tomando en consideración los siguientes componentes:

- La acción a realizar por el alumno con el contenido que debe asimilar

- Las condiciones en que el alumno debe realizar la acción.

- Los indicadores cualitativos de la acción a formar.

De aquí resulta que el objetivo queda expresado en función de las habilidades de representar, leer e interpretar documentación técnica gráfica, según sea el caso, aplicando el sistema operacional de la habilidad a lograr.

\section{b) Modelos de los contenidos}

Una de las necesidades actuales del conocimiento científico y de la actividad práctica consiste en la capacidad del individuo para pensar de forma sistémica, de manera que posibilite la solución exitosa de muchos problemas de su actividad profesional, siendo necesario que los contenidos se expresen en términos de sistemas de conocimientos, sistema de habilidades y sistema de valores. 
En la concepción de la asignatura se parte de un enfoque estructural funcional con carácter de sistema. Estructurándose la asignatura en tres temas que integran los contenidos del Dibujo con los contenidos de la Topografía y estando los mismos relacionados, lo que revela que cada nivel del sistema se estructura y tributa a la formación de las habilidades objeto de estudio, como núcleo central del sistema.

La estructuración propuesta posibilita una organización lógica del pensamiento que contribuye en grado significativo al desarrollo de las referidas habilidades en los alumnos de Agronomía, lo que le permitirá resolver diferentes tareas de carácter técnico en su vida profesional, basado en los planos y mapas topográficos, los cuales constituyen un instrumento de trabajo fundamental en su profesión.

Para contribuir al desarrollo de las habilidades de representación lectura e interpretación de documentación técnica gráfica se debe tener en cuenta que, en el aprendizaje y durante el proceso de asimilación del conocimiento se ejecutan acciones propias de la asignatura y acciones propias del nivel de desarrollo del pensamiento lógico, siendo necesario establecer las relaciones correspondientes entre ellas de manera tal que durante la ejecución y ejercitación de acciones operacionales se logren dichas habilidades.

\section{Base Orientadora de la Acción (BOA) y etapa materializada}

En esta propuesta se presenta la etapa de formación de la BOA y etapa materializada. Para ello se tiene en cuenta:

- Selección de los procedimientos y operaciones objeto de asimilación en el modelo del contenido.

- Diseño de la BOA propia para cada procedimiento.

- $\quad$ Análisis de los métodos factibles para conformar la BOA.

- $\quad$ Sistema de tareas para llevar a cabo la etapa materializada.

En el diseño de la BOA de cada procedimiento se trabaja teniendo en cuenta que el grado de despliegue abarque la mayor cantidad de detalles posibles, lo que contempla las operaciones conformadoras de cada acción con vistas al logro de las referidas habilidades.

Para conformar esa base orientadora generalizada y completa, se sugiere la participación activa y consciente (al menos a nivel de comprensión de esa base) del alumno, debe apropiase de la lógica de la formación del procedimiento como paso previo al tránsito hacia la etapa materializada. Por esta razón, la aplicación de métodos de la enseñanza problémica (en nuestra propuesta a través de la enseñanza 
en Taller), puede resultar apropiado para desarrollar esta etapa, donde la orientación esté presente durante la formación de la BOA. (Pérez, 1998, p. 5)

\section{Aparato cognitivo de la metodología}

\section{Los principios de la metodología}

Los principios que sustentan la metodología son los aportados por la ciencia pedagógica y sistematizados por la autora Díaz (2005, p. 28).

1. Principio de carácter sistémico para el diseño del proceso enseñanza - aprendizaje. Este principio se revela en la concepción del contenido de las habilidades de expresión gráfica; en el establecimiento de la interrelación de las acciones y etapas de la metodología, teniendo en cuenta los diversos grados de complejidad a que debe enfrentarse el alumno en formación de lo más fácil a lo más complejo; en la integración de las operaciones de las referidas habilidades.

2. Principio de carácter contextualizado para el diseño del proceso de enseñanza aprendizaje. La metodología se sustenta en el principio de que el contexto en que se desarrolla el proceso de enseñanza - aprendizaje desempeña un rol fundamental como punto de partida para el alumno en formación a partir de los contextos del grupo, la escuela, la familia y la comunidad como fuentes del contenido y agentes socializadores del aprendizaje.

3. Principio de carácter personológico para el diseño del proceso enseñanza aprendizaje. Parte de que cada alumno en formación esté preparado para asimilar el contenido objeto de aprendizaje, lo asuma desde su individualidad, no como norma rígida, sino con carácter flexible, lo que le permite incorporar a la actividad de aprendizaje, sus experiencias, aprovechando sus potencialidades, individuales, compensando sus limitaciones y superándolas.

4. Principio de carácter interdisciplinario para el diseño del proceso enseñanza aprendizaje. En este principio se pone de manifiesto la necesidad de que en el diseño del proceso enseñanza - aprendizaje se asuma con un enfoque integrador de la formulación de los objetivos en cada uno de los temas de la asignatura, en la selección de los contenidos y de las fuentes de información con una concepción interdisciplinaria, en la concepción de formas organizativas que permitan dar tratamiento a contenidos de diversas disciplinas y en el carácter integrador y formativo de la evaluación. 
5. Principio de carácter planificado del proceso enseñanza - aprendizaje. Se revela en la necesidad diseñar la actividad docente para lograr efectividad en el proceso de formación integral de los alumnos, teniendo en cuenta el diagnóstico pedagógico y las condiciones para su realización, las formas organizativas, las metas a alcanzar a largo y corto plazo. Asumir este principio en la metodología propuesta condiciona el carácter de dirección que debe poseer la actividad docente.

\section{Principales definiciones del cuerpo categorial de la metodología}

Para llevar a efecto el proceso de enseñanza - aprendizaje de la asignatura Dibujo Topografía, se propone utilizar como estrategia la aplicación de la Computación Gráfica a través de los recursos que nos brindan estas novedosas técnicas y los métodos activos de enseñanza - aprendizaje.

Si en la década del 80 del pasado siglo, el desarrollo de la informática era limitado y la infraestructura del país permitía una gran diversificación de especialidades y especializaciones en el campo de las ingenierías, con un perfil estrecho y con un campo de conocimiento limitado, hoy es diferente. Se necesita de un profesional de perfil ancho con una amalgama de conocimientos que permita entender primero la realidad que enfrenta y segundo, proporcionarle las herramientas para que sea capaz de interpretar cada fenómeno y tenga la visión necesaria para proponer o darle adecuada solución.

La era informática ha revolucionado todas las áreas del conocimiento humano y el Dibujo no ha escapado a su influjo. A partir del año 1962 es que el Dibujo adquiere una dimensión interactiva, cuando en el Instituto de Tecnología de Massachussets, un joven llamado Iván Sutherland sentó las bases de lo que conocemos hoy como Gráficos o Imágenes interactivas por ordenador. Este brillante alumno en su tesis doctoral titulada, " Sketchpad: A Man - Machine Graphic Comunications Sistem," propuso la idea de utilizar un teclado y un lápiz óptico para crear e interactuar con gráficos en la pantalla del monitor.

La estructura de datos utilizada por Sutherland en la computadora TX-2, se basaba en la topología del objeto que iba a representar, describiendo con exactitud las relaciones entre las partes componentes del mismo, introduciendo los elementos generadores de lo que hoy se conoce como Programación Orientada al Objeto. Con el primer programa de Gráficos por Computadora u Ordenador, nacía la Informática Gráfica, en el Instituto de Tecnología de Massachussets. A su vez, las Ciencias Técnicas en su "simbiosis" con la Informática, han Volumen 8, Número 3, Año 2008, ISSN 1409-4703 
dado lugar a la joven computadora y a software cada vez más abarcadores e inteligentes, se ha ido materializando aquel sueño de Dibujar y Diseñar gráficamente por computadoras. El Dibujo adquiere una nueva dimensión con el desarrollo de la Informática o Computación, lo que sin lugar a dudas, lo sitúa en planos cualitativos superiores.

El 7 de marzo de 1991, el Presidente Fidel Castro dejó inaugurado el palacio de la computación en Ciudad de La Habana, estaba materializando la sabia decisión de estimular y apoyar en todo lo posible el proyecto de informatización de la sociedad cubana; a partir de ese año se inició para Cuba una nueva era, que se refleja en la visión estratégica de que "el futuro será un eterno Baraguá"; a esta se añadió luego la consigna de "Ganar esta guerra a pensamiento", (citado por Murciego, 2004:!5). La importancia trascendental de los conceptos que encierran ambas ideas, constituyen la fuente de inspiración para no decaer en el empeño de continuar en la materialización práctica de este proyecto de informatización de la sociedad cubana.

\section{La Computación Gráfica}

La Computación Gráfica abarca un campo que se ocupa de toda la Tecnología de Gráficos y sus aplicaciones. La CG combina aspectos teóricos y prácticos en función de la comunicación o la solución de problemas de otras ciencias particulares y en especial de las Ciencias de la Ingeniería.

"A este respecto, plantean los Profesores J. R. Lama Ruiz; F. Aguayo González y N. del Pozo Madroñal, del Departamento de Ingeniería del Diseño de la Universidad de Sevilla, lo siguiente: Podríamos caracterizar esta área del conocimiento, por su Objeto de estudio, por sus $\underline{\text { Técnicas y procedimientos, así como por su Intencionalidad cognoscitiva. }}$

Objeto de estudio. El objeto de estudio es el MODELADO icónico, estático y dinámico de estructuras, funciones referidas a sistemas naturales y artificiales, con propósitos creativos, constructivos o comunicacionales.

Técnicas y procedimientos. Estas son muy diversas, cabe citar las siguientes:

- Técnicas clásicas de geometría descriptiva, que algunos autores han denominado geometría constructiva.

- Técnicas clásicas de sistemas de CAD.

- Técnicas de animación. 
- Técnicas de síntesis de escenarios virtuales multimedia.

- Técnicas de modelado formal-simbólico como instrumento mediacional, para el modelado icónico computacional.

La intencionalidad cognoscitiva se ciñe a la dimensión de "conocer para hacer" en el ámbito técnico-constructivo y comunicacional, sin abandonar el terreno de la visualización científica que aparece en problemas técnicos constructivos o funcionales" (Morciego, 2004, p.91).

En Cuba, el Área de Expresión Gráfica abarca convencionalmente un conjunto de asignaturas y materias cuyos límites se entrelazan e imbrican con otras ciencias y áreas del conocimiento en una interrelación dialéctica que la hace más rica y dinámica. Algunas de las materias que la componen actualmente son: Geometría Descriptiva, Dibujo Básico, Dibujo Aplicado Diseño Gráfico Asistido por Computadora (conocido como CAD), Topografía, Cartografía, etc.

\section{Los métodos activos de enseñanza}

Desde hace algún tiempo en la enseñanza superior en Cuba, se viene enfatizando en la urgente necesidad de la utilización de los métodos activos de enseñanza como vía única para la preparación de un profesional capaz de enfrentar los retos que le impone la profesión en el entorno donde se desenvuelve, dados los grandes avances de la ciencia y la tecnología, cambios en el ámbito nacional e internacional, que en la última década han tenido lugar. Por un lado la globalización neoliberal que nos afecta y nos obliga a ser más competitivos para sobrevivir en este apocalíptico mundo y por otro lado las dificultades financieras, el deterioro del medio ambiente, etc.

Nadie puede imaginar que con una preparación tradicional de los alumnos, en el que el papel fundamental lo realiza el docente, pueda lograrse tal capacidad. Ello requiere del trabajo independiente del alumno que se logra a través de los métodos activos de enseñanza, que según algunos autores, son aquellos que conciben el proceso de enseñanza - aprendizaje como un todo integrado, en el que se pone de relieve el papel protagónico del alumno, revelándose como característica determinante la integración de lo cognitivo y lo afectivo, de lo instructivo y lo educativo, como requisitos pedagógicos y psicológicos esenciales. 
Diferentes autores han hecho diversas clasificaciones sobre los métodos activos, pero lo cierto es que los llamemos de una forma u otra, los métodos activos son todo lo que el docente logre hacer en una clase, de forma tal que la misma sea lo suficientemente activa para lograr desarrollar en los alumnos la creatividad y la independencia cognoscitiva.

\section{Conceptualización de las habilidades, campo de estudio y sus sistemas operacionales}

Se define la habilidad "como el nivel de dominio de la acción en función del grado de sistematización alcanzado por las operaciones correspondientes; es decir, para reconocer la presencia de una habilidad es necesario que en la ejecución de la acción se haya logrado un grado de sistematización tal, que conduzca al dominio del sistema de operaciones necesarias, imprescindibles y esenciales para su realización. Desde la Didáctica se asume que la habilidad es el componente del contenido que caracteriza las acciones que el estudiante realiza al interactuar con el objeto de estudio: el conocimiento". (Montes de Oca, 2001, p. 32)

"Habilidad representar gráficamente. Permite dejar constancia proyectiva de la forma de un objeto de carácter material o ideal, mediante una codificación establecida de acuerdo a las leyes de la Teoría General de las Proyecciones lo que lleva implícito la acción de proyectar" (Pérez, 1998, p. 53).

\section{Sistema operacional:}

>Analizar los datos tomados del registro de campo y de los cálculos.

$>$ Seleccionar el instrumental y medios necesarios para realizar el dibujo.

DElegir la escala a la que se va a realizar el dibujo.

- Realizar las conversiones de las medidas reales a gráficas de acuerdo a la escala elegida.

$>$ Elegir el formato que se va utilizar de acuerdo a las normas técnicas.

> Seleccionar los símbolos convencionales que se van a utilizar de acuerdo a las normas técnicas establecidas.

Decidir el rotulado técnico (tamaño de letras y números por utilizar).

Elaboración del plano.

“Habilidad lectura. Consiste en la decodificación integral del objeto proyectado. Lleva implícita la acción identificar. 


\section{Sistema operacional:}

$>$ Observar cuidadosamente el plano o mapa en toda su extensión

$>$ Identificar los diferentes elementos y partes que contiene el plano, como son: escala (numérica y gráfica), símbolos convencionales (de relieve, de vegetación, de agua y de construcciones).

>Analizar la posición de los objetos representados en el plano en relación a la orientación de estos, teniendo en cuenta la red de coordenadas (meridiana y paralela).

$>$ Identificar y caracterizar las diferentes formas del relieve del terreno de acuerdo a su representación por curvas de nivel” (Paz,2008: 45)

Habilidad interpretación. Consiste en asignarle significado a las proyecciones, de manera que adquieran sentido en función del propio objeto. Tiene implícito la acción abstraer (Pérez, 1998:54).

Sistema operacional:

$>$ Logra una abstracción mental de lo leído

> Separar mentalmente las cualidades del objeto para considerarlo aisladamente.

$>$ Establecer la relación con los conocimientos anteriores y el fenómeno objeto de interpretación.

$>$ Determinar las relaciones causa - efecto.

\section{Elementos del aparato instrumental de la metodología}

El aparato instrumental está compuesto por los procedimientos del docente y del alumno para el desarrollo de las habilidades de expresión gráfica y la planificación de la instrumentación de la metodología en correspondencia con el aparato cognitivo.

Procedimientos a ejecutar por el docente que dirige el proceso de enseñanza - aprendizaje y del alumno en formación para el desarrollo de las habilidades de expresión gráfica.

\section{Procedimientos a ejecutar por el docente que dirige el proceso de enseñanza - aprendizaje}

La orientación del docente está presente durante todo el proceso de enseñanza aprendizaje y debe propiciar un clima socio - psicológico afectivo y participativo, que se caracterice por una comunicación fluida, auténtica, libre, solidaria, respetuosa, estimulante, que revele confianza en los estudiantes para que puedan llegar a alcanzar niveles excelentes en el dominio de los sistemas de operaciones y así lograr un desarrollo más Volumen 8, Número 3, Año 2008, ISSN 1409-4703 
eficiente en la ejecución de las habilidades de expresión gráfica. Para lograr dicho propósito, es necesario que en las diferentes actividades organizativas del proceso docente se exprese una orientación general de los contenidos, los procedimientos e indicadores que permiten asumir la base orientadora para su ejercitación, precisar una orientación más personalizada para que el alumno en formación ejecute las tareas docentes con un enfoque profesional que les permita el desarrollo de dichas habilidades.

1. Diagnóstico del estado de desarrollo del nivel computacional de los alumnos en formación.

Para diseñar y aplicar instrumentos de diagnóstico el docente debe partir de los objetivos, las dimensiones e indicadores establecidos para el año en la carrera, en relación con el desarrollo de las habilidades de expresión gráfica. Los instrumentos deben tener en cuenta el estado de la práctica pedagógica en relación con esta problemática y lo que está orientado por el MES. Se debe establecer la relación entre el nivel computacional de los alumnos y la asignatura que van a recibir (Dibujo - Topografía), donde van a aplicar la computación gráfica.

Precisar los resultados del diagnóstico a partir de las dimensiones e indicadores del año significa que el docente identifica el estado de desarrollo de las habilidades y conocimientos computacionales que tiene el alumno para enfrentarse a la asignatura de Expresión Gráfica a través de la enseñanza asistida por computadora.

2. Proyección del programa que se imparte hacia el desarrollo de las habilidades de expresión gráfica en correspondencia con los objetivos de año.

A partir del diagnóstico el docente que dirige el proceso de formación inicial debe proyectar el diseño de su programa e incorporar los cambios necesarios en función de lograr las transformaciones que permitan el desarrollo de las habilidades de expresión gráfica en los alumnos de Ingeniería Agronómica.

El docente realiza un profundo estudio de su programa, identifica las potencialidades que este aporta al desarrollo de las habilidades de representación, lectura e interpretación de documentación técnica gráfica en estrecha relación con el diagnóstico y los objetivos para el año.

El docente, como organizador y guía del proceso de enseñanza - aprendizaje, debe ser un observador sistemático de cada estudiante en formación, en el desarrollo de las habilidades 
mencionadas, para orientar de forma adecuada el proceso operacional y de acciones con vistas a lograr el desarrollo más eficiente de las habilidades de expresión gráfica.

3. Orientación del sistema de tareas docentes que contribuya al desarrollo de las habilidades de representación, lectura e interpretación de documentación técnica gráfica. Al proyectar el sistema de tareas docentes por temas en el programa de la asignatura, se deben incluir los ejercicios con enfoque profesional pedagógico a partir del vínculo con la práctica, que permita la ejercitación sistemática de los pasos de la estructura interna del proceso docente - educativo, de acuerdo a las características del año, que conducirán al desarrollo de las habilidades de expresión gráfica en el futuro ingeniero. En estas tareas docentes se explica la base orientadora de la actividad según las necesidades de los alumnos en formación y las diferentes situaciones pedagógicas en las que se manifieste en cada tema y en el trabajo final integrador de la asignatura.

4. Control y evaluación del desarrollo de las referidas habilidades alcanzado por los educandos en formación

La evaluación se asume en la metodología como un componente del proceso de enseñanza que permite regular y estimular la asimilación de los contenidos conceptuales, procedimentales y actitudinales necesarios y suficientes para alcanzar los niveles de desarrollo de las habilidades de expresión gráfica en el proceso de enseñanza - aprendizaje de la asignatura. Se sistematiza en todas las actividades de aprendizaje que se desarrollan en el proceso de formación y tienen una connotación especial para evaluar el desempeño alcanzado por los alumnos en formación, en el Trabajo Final Integrador de la asignatura.

Desde la formulación del objetivo se está diseñando la propia evaluación; también las actividades de aprendizaje constituyen instrumentos de evaluación, además se diseñan otros instrumentos con objetivos precisos para medir niveles de integración del contenido y diagnosticar el desarrollo integral de los alumnos a partir de la asimilación del contenido. Los instrumentos que se utilicen deben permitir evaluar todas las dimensiones de la formación de la personalidad del alumno, en los que se revelen los componentes conceptuales, procedimentales y actitudinales. El docente selecciona, además, las técnicas que se utilizarán, las formas, el tiempo disponible y las condiciones para su aplicación.

La evaluación debe estar dirigida fundamentalmente a valorar la calidad del proceso, por lo que las técnicas deben permitir la valoración de la actuación de los alumnos durante la 
actividad y no solo de los resultados finales, de tal manera que los docentes y los alumnos puedan reconocer los problemas y las causas que originan las limitaciones en el aprendizaje y encontrar las vías para darle solución. En tal sentido es recomendable la introducción de técnicas de coevaluación y la autoevaluación entre los alumnos.

Un componente importante de diseño de la evaluación es la determinación de los indicadores para cada contenido y la escala para la valoración de su calidad, cuestión que debe ser discutida con los alumnos. Es necesario incluir como indicadores las habilidades que desarrolla el alumno en el manejo y utilización de las principales fuentes de información tales como: el libro de texto, los software educativos, modelos tridimensionales, maquetas, planos, y el equipamiento "tradicional" de Topografía y Dibujo Técnico. (Díaz, 2005:55).

\section{Procedimientos a ejecutar por el alumno en formación}

1. Orientación en las características y la manera de alcanzar el desarrollo de las habilidades expresión gráfica.

El alumno en cada actividad docente debe:

- Analizar las características de la tarea docente planteada.

- Valorar su interrelación con las habilidades de expresión gráfica.

- Ejecutar la acción con vistas a lograr dichas habilidades.

2. Ejecución del sistema de tareas docentes de la asignatura y su integración, al finalizar cada tema.

El alumno debe:

- Reflexionar acerca de los pasos que deben tenerse en cuenta para ejecutar la instrumentación propuesta.

- Ejecutar la instrumentación de acuerdo con los indicadores planteados por el profesor en el Trabajo de Control Extraclases (TCE).

3. Auto evaluación del nivel de desarrollo alcanzado en el desarrollo de las habilidades de representación, lectura e interpretación de documentación técnica gráfica.

El alumno elaborará las conclusiones cognitivas relacionadas con el desarrollo de las habilidades de expresión gráfica planteadas en la tarea docente (Bermúdez, 1996). 


\section{Conclusiones}

- En esencia se plantean los fundamentos teóricos de una metodología basada en métodos activos de enseñanza -aprendizaje y medios de enseñanza, apoyados en la computación gráfica, capaces de que en menos tiempo de actividad docente presencial se logre una docencia en la asignatura Dibujo - Topografía de alta calidad, que contribuya a satisfacer las necesidades actuales del egresado en Ingeniería Agronómica en cuanto al desarrollo más eficiente de las habilidades de representación, lectura e interpretación de documentación técnica gráfica. A la vez se pretende que la propuesta contribuya a que el futuro profesional de esta especialidad asuma de forma consciente su modo de actuación a partir de sus propias características, respondiendo a fines y objetivos sociales en correspondencia con su responsabilidad ante la sociedad.

- La propuesta metodológica que se propone responde a un proceso de enseñanza aprendizaje desarrollador, productivo, creador, portador de lo instructivo - educativo, promotor o agente del cambio educativo, de lo afectivo - cognitivo, condicionada de motivaciones intrínsecas, y de la comunicación interpersonal, entre otros aspectos significativos y desarrolladores.

- La eficiencia y éxito del proceso de enseñanza - aprendizaje de la asignatura Dibujo Topografía a través de esta propuesta metodológica está dado porque plantea el desarrollo e independencia de la actividad cognoscitiva y creadora de los alumnos y el papel dirigente del docente. 


\section{Referencias}

Díaz González, Lourdes. (2005). Metodología para desarrollar las habilidades de diseño del proceso pedagógico en la formación inicial del profesor general integral de Secundaria Básica. Villa Clara, Cuba. Tesis Doctoral. ISPFV.

Montes de Oca, N. (2004). La formación y desarrollo de habilidades en el proceso docente. Universidad de Camagüey, Cuba. Monografías.

Morciego, Carlos. (2004). Historia de la Geometría Descriptiva y el Dibujo Técnico en Cuba. Rosario, Argentina Trabajo publicado en www.ilustrados.com. EGRAFIA, 2004.Primer Encuentro Internacional de profesores e investigadores del área de sistemas de representación.

Paz, Oscar. (2008). Metodología para el desarrollo de las habilidades de expresión gráfica en los estudiantes de Ingeniería Agronómica de la Universidad de Ciego de Ávila. Tesis Doctoral. ISPMAD. Ciego de Ávila. Cuba.

Pérez, María Cristina. (1998). Sistema de procedimientos algorítmicos y propuesta metodológica para su tratamiento en la geometría descriptiva. Málaga, España. Actas del X Congreso de Ingeniería Gráfica 3 t. en 3 v. 